\title{
Numerical Analysis of the Dynamic Response of Slope under the Blasting Vibration Effect
}

\author{
Iau Teh Wang
}

\begin{abstract}
The effect of blast-induced seismic effect on slope can be considered as a transferred and transformed process of the energy. It is necessary to know the attenuation law of blast-induced seismic wave, in order to make relatively accurate estimation for the extent of the slope affected by blasting vibration. This study applied the LS-DYNA software and adopted the Arbitrary Lagrangian-Eulerian method for numerical analysis. Simulate the surface blast of $\mathrm{C}-4$ explosives in a semi-infinite space, in order to explore the slope stability with different height and gradient are computed. The result shows that the propagation law and attenuation characteristics of the detonation earthquake wave on slope are different to the condition of level ground. Comparing the horizontal displacement and vertical acceleration of the top and toe of the slope, vertical acceleration of the top particles are larger then those of toe, so there is vibration amplification effect at the top of the slope. The slope crest displacement changes slightly decrease when the slope height reaches a certain degree. The attenuation effect of blast-induced seismic wave and the amplification effect of slope exist at the same time. This study can be used as a reference in hazard control of explosion-induced vibration for the structures on the slope.
\end{abstract}

Index Terms-Blasting, seismic wave, vibration effect, surface acceleration, slope.

\section{INTRODUCTION}

Slopes are formed naturally or artificially. They are also one of the primary geological environments used in project construction activities. Subsequently, explosion techniques are employed during project construction to reduced construction time and save investment costs. However, seismic effects induced by blasts are extremely likely to damage slopes. The influences that blast-induced seismic effects have on slope stability have gradually become prominent problems, and because explosion-induced seismic effects commonly cause numerous disasters and accidents, such as collapses and landslides, the sediment production in catchment areas are subsequently effected. Thus, researching the stability of slopes under varied blast-induced dynamic loads is a significant topic that should be carefully addressed prior to construction planning and design, and the strengthening of this research field has become increasingly urgent. This study primarily explored the influences that blast-induced seismic effects have on the soil properties of slopes. By investigating the propagation patterns of blast

Manuscript received November 26, 2013; revised March 14, 2014. This study was partially supported by the National Science Council in Taiwan R.O.C. under through Grant NSC 102-2218-E-145-001.

Iau Teh Wang is with the Department of Civil Engineering, R.O.C Military Academy, Fengshan, Kaohsiung, CO 83059 Taiwan R.O.C. (e-mail itwangroc@gmail.com). vibrations, the dynamic responses of slopes under blast loads, as well as the related influences, can be identified and confirmed. With these results, the production of blast-induced seismic energy can be reduced, further decreasing blast-induced ground vibration hazards and maintaining slope stability.

The blast of explosives detonated in soil or rock material is transferred outwards in the form of waves by passing through related media. In the transmission process, a portion of the energy is converted into seismic waves. These seismic waves induce the particles in the medium to vibrate, thereby causing the ground to vibrate. The ground vibrations generated by blast-induced seismic waves are known as blast vibrations. When blast-induced vibrations reach a specific strength, construction facilities and surrounding environment within the influence range sustain varying levels of negative influences. These influences are known as seismic effects [1].

When explosives are detonated on land surfaces or at low-altitudes, seismic waves are primarily stimulated by the air blast; the waves first propagate underground and then generate vibrations in the surface particles [2]. The blast-induced shock waves in the air compress the land surface (known as soil compressive waves), which consequently converts into elastic seismic waves. Therefore, during surface blasts, both types of blast waves can be measured inside the soil [3]. That the air blast near the explosion point is fast and strong, and the velocity and strength of corresponding soil compressive waves consequently increased. This is the key parameter that influences ground vibration [4].

Researched the influences that the blast operations in the mining area of Eastern Taiwan have on slope and moldering wall stability. Shen's analytical results indicated that the influences and damages that blast vibrations have on slopes and final moldering walls can be evaluated using the physical quantities of vibration velocity, acceleration, stress, and displacement [5], [6]. Current blast projects investigate blast vibration parameters based on the concepts of blast energy conversion and the theories of mass, energy, and momentum conservation when blast and shock waves are transmitted. Based the aforementioned construction characteristics, the influences that blast vibrations have on the effects and damage of the protected targets can be evaluated using the physical quantities of vibration velocity, acceleration, stress, and displacement. Project construction subsequently uses the vertical peak particle vibration velocity or acceleration as a reference for blast vibration strength. Thus, analyzing the hazard degree of near-surface or surface blasts is primarily based on the transmission properties of air blast waves and the vibration strength of land surfaces. 
The primary influence that blast-induced seismic effects have on slope stability is the reduction of shear strength with the increase of inertial force. Following this influence is the continuous vibration effect. This effect causes an increase in shearing stress within the slope body, deteriorating the original cracks within the native land structure and simultaneously generating new blast cracks; thereby influencing slope stability. Baker and Garber (1978) [7] proposed a static calculation method to evaluate the stability of slope under dynamic loads. Cherles (1988) [8] used a discrete element model to investigate blast vibrations at different frequencies and analyze 2D rock-based slope stability. The results indicated that vibration frequency influences slope stability. Ma et al. (2000) [9] employed software to simulate the transmission conditions of blast waves in the soil, investigating the spectral properties of blast waves and their effects on the building structures.

Most analytical studies related to blast vibrations can roughly be divided into experimental and numerical simulation analyses [10]-[12]. Blast experiments require substantial costs, exhibit location limitations, and possess a certain level of danger, limiting blast-related on-site experiments. Thus, to understand the effects that blast-induced seismic effects have on slope stability, this study considered the attenuation of blast energies during surface contact explosions and the propagation properties of blast waves in slopes following changes in time and space. This study further adopted the LS-DYNA software as the analytical tool to confirm the dynamic slope responses during blasts, thereby reducing the generation of blast-induced seismic energy and decreasing hazards caused by blast-induced ground vibrations. The results of this study can be provided as a reference to slope construction structures in their anti-blast vibration designs.

\section{RESEARCH METHODS}

This study adopted the Arbitrary Lagrangian-Eulerian (ALE) numerical finite element method, along with the application of the LS-DYNA software to comprehensively describe the hydrodynamic behaviors of blast-generated gases.

This method effectively analyzes the interaction effect of gases and solids and further solves dynamic analysis issues, such as geometric non-linearity, material non-linearity, and contact non-linearity. Subsequently, the US military compliant empirical equations, TM5-1300 (1990) [13] was used to cross-verify the reliability of the simulations.

\section{A. Basic Theory of Fluid-Structure Interaction}

The methods to describe hydrodynamics and non-linear solid mechanics numerical simulation analyses include three numerical description methods, specifically, the Lagrangian, Eulerian, and ALE methods [14].

The advantages of the Lagrangian method is the ability to accurately describe the motion of structural boundaries and track free surfaces or interfaces of varying materials. However, the disadvantage is that serious grid distortion occurs following major deformation, further affecting the solution process or even terminating numerical computations.
Subsequently, the advantage of the Eulerian method is the ability to easily process major deformations. However, this method is comparatively inferior in capturing structural boundaries and requires excessive computational times.

The ALE method simultaneously contains the advantages of both the Lagrangian and Eulerian methods. The Lagrangian method is applied to effectively track the motion of structural boundaries. ALE further uses the advantages of the Eulerian method in terms of grids. However, the main difference between the Eulerian method and ALE is that the latter method can freely shift or adjust spatial grids based on the pre-defined parameters during the solution processes. Thus, the ALE method initially executes one or several Lagrangian grid computations. The grid unit begins to deform following material movement. Subsequently, the ALE method executes the time-step computations. Through these computational procedures, ALE can effectively handle fluid-structure interaction issues. ALE description resolves the individual drawbacks of Eulerian and Lagrangian descriptions and can handle problems such as explosions and high velocity impact [15], [16].

ALE description and solved the mass conservation equation, momentum conservation equation and energy conservation equations, as in (1), (2), (3); When the mesh velocity is $0\left[v_{w}(t)=0\right]$, it is a Eulerian description; when the mesh velocity equals the material velocity $\left[v_{w}(t)=u\right]$, it is a Lagrangian description; otherwise, the equations are between Eulerian and Lagrangian descriptions [17].

$$
\begin{gathered}
\frac{d}{d t} \int_{s(t)} p d v=-\int_{\partial s(t)} \rho\left(u-v_{w}\right) \cdot n d s \\
\frac{d}{d t} \int_{s(t)} p u d v=-\int_{\partial s(t)} \rho u\left(u-v_{w}\right) \cdot n d s-\int_{s(t)} \nabla \cdot p d v \\
\frac{d}{d t} \int_{s(t)} p I d v=-\int_{\partial s(t)} \rho I\left(u-v_{w}\right) \cdot n d s-\int_{s(t)} p u \cdot p d s
\end{gathered}
$$

where $S(t)$ is an active area in space, $\partial s(t)$ is its boundary and $v_{w}$ is the velocity of $\partial s(t)$.

\section{B. Element Type and Time Integration}

Blast vibration analysis involves transient dynamics issues. The differential equations are related to the derivatives of time and space, and the numerical simulations must effectively handle time integration. This type of integration method can further be divided into explicit time integration and implicit time integration. The explicit method utilizes pre-determined moment solutions to solve following moment solutions; conversely, the implicit method uses the iterative method to obtain solutions.

Typically, Interval $\Delta t$ employed in the explicit time integration method requires small time increments to prevent the occurrence of computational errors. The LS-DYNA program is primarily based on explicit time integration, which acts as a type of conditional stability. The numerical integration method is based on two basic concepts [18]: (1) the motion equation can only be satisfied when being discrete 
on a specific Time Interval $\Delta t$ point; and (2) the overall time integration is based on the approximate solution of individual time when the content of the Time Interval $\Delta t$, including the changes in displacement, velocity, and acceleration, is segmented into identical $n$ segments between Time $t_{j}$ and $t_{j+n}$; in addition, the solution of any Time $t$ is the initial condition of the following Time $t+\Delta t$.

In this study, the analysis is done by using the ALE method with three-dimensional eight-node (8-Node) solid elements. Elements are defined as 8-Node. In $\mathrm{X}, \mathrm{Y}$ and $\mathrm{Z}$ directions, there is a degree of freedom in translation. Together with degrees of freedom in velocity and acceleration, there are in total nine degrees of freedom for each node. The shape function of 8-Node solid elements is defined as in (4) [19]; Fig. 1 is the demonstration of a solid element.

$$
\phi_{j}=1 / 8\left(1+\xi \xi_{j}\right)\left(1+\eta \eta_{j}\right)\left(1+\xi \xi_{j}\right)
$$

where $\xi_{j}, \eta_{j}$ and $\xi_{j}$ are the natural coordinates of the unit, and depending on the location, the node values are $\pm \mathbf{1}$.

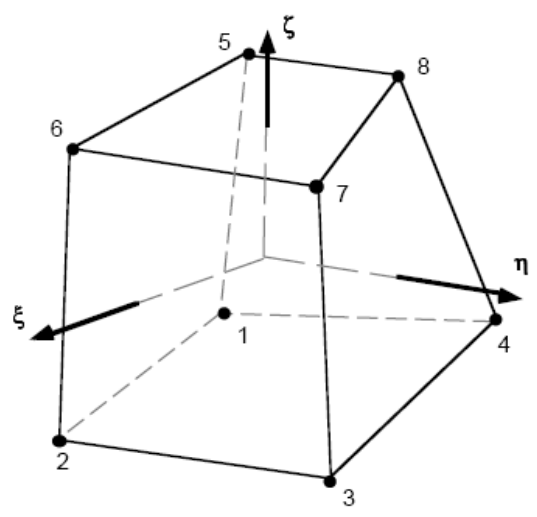

Fig. 1. Demonstration of a solid element. [19]

In the finite element method, $\Delta t$ is related to the geometric conditions of elements and material velocity. The stability conditions of $\Delta t$ for 8-Node Solid Element are as in (5);

$$
\Delta t=\frac{L_{e}}{\left\{\left[Q+\left(Q^{2}+c^{2}\right)^{1 / 2}\right]\right\}}
$$

The wave propagation velocity of regular elastic materials is defined as in (6):

$$
c=\sqrt{\frac{E(1-v)}{(1+v)(1-2 v) \rho}}
$$

where $L_{e}=v_{e} / A_{e \max } ; Q$ is the bulk viscosity coefficient; $L_{e}$ is a characteristic length; $v_{e}$ is the element volume; $A_{e_{\max }}$ is the area of the largest side; $c$ is the material wave velocity; $\rho$ is the specific mass density; $E$ is Young's modulus; and $v$ is Poission's ratio.

\section{NUMERICAL ANALYSIS}

The purpose of this study is to investigate the influences that blast-induced seismic effects when C-4 explosives detonate on land surfaces. Primarily, the degree of influence that blast vibration strength has on the gradient and height of slopes under dynamic loads was determined. Complemented by the LS-DYNA analytical tool, the fluid-structure interaction method was used to simulate blast experiments. The explosives and air were set into Eulerian grids and the soil was set into Lagrangian grids to establish a fluid-structure numerical analysis model. This model was then used to analyze the blast-induced shock waves and the land surface acceleration.

\section{A. Analytical Model}

This study aimed to investigate the influences that blast-induced seismic effects have on slopes and analyze the degree of influence on the gradient and height of the slopes under varying dynamic loads. The analytical model was established using an eight-node solid element model. The units were globally set as cm-g- $\mu$ s. The boundaries were set as non-reflective boundary simulations blasting in an infinite area. The explosives were rectangular C-4 with a weight of 5 pounds $(2,200 \mathrm{~g})$ and a density of $1.601 \mathrm{~g} / \mathrm{cm}^{3}$. The dimensions of the explosives were $10 \times 5 \times 28(\mathrm{~cm})$. The explosives were placed in the center of the model in contact with the land surface. The detonations were stimulated at close range $(50 \mathrm{~cm}$ from the toe of the slope) to determine the influences that the blasts have on slope stability.

Concerning the slope numerical model, the slope was fixed at $45^{\circ}$ to determine the influences at varying heights $(\mathrm{cm})$ of $100,150,200,250$, and 300. Subsequently, the height of the slope was fixed at $300 \mathrm{~cm}$ to determine the influences of at varying gradients of $30^{\circ}, 45^{\circ}, 60^{\circ}$, and $75^{\circ}$. A $1 / 2$ model symmetry was used and the finite element grid sizes of air, explosives, and slope were $5 \mathrm{~cm}, 5 \mathrm{~cm}$, and $10 \mathrm{~cm}$, respectively. The analytical model is illustrated in Fig. 2 and Fig. 3.

The minimum time interval of the element, i.e., the smallest mesh unit is used in the solving process and determines the calculation accuracy, stability and computation time. For stability, usually $\alpha \leq 0.9$ is used. In explosion simulations, $\alpha \leq 0.67$ is used [20].

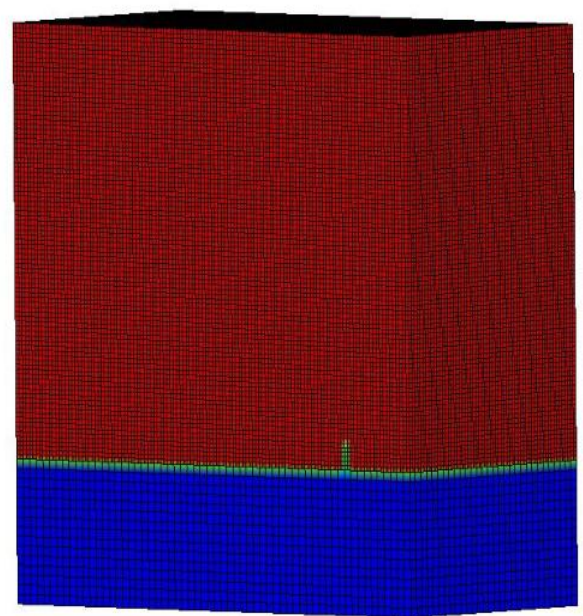

Fig. 2. Free field pressure analyzes of $1 / 2$ symmetry model. 


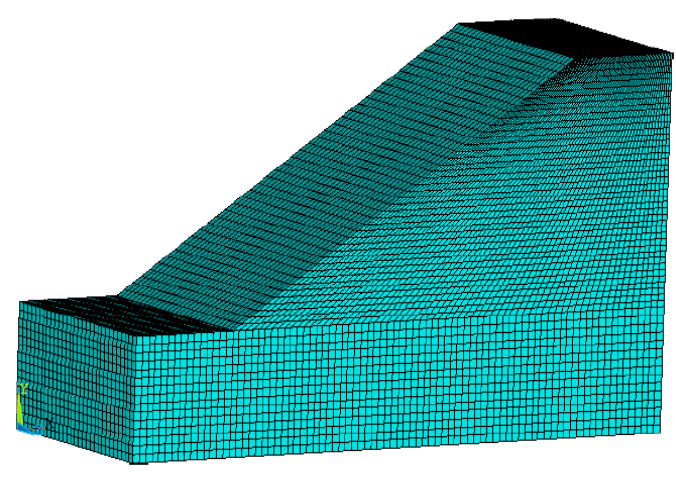

Fig. 3. Slope analyzes of $1 / 2$ symmetry model.

\section{B. Explosive Parametes and Equation of State}

The MAT_HIGH_EXPLOSIVE_BURN material model was used to simulate the high explosive model. Equation (7) is the JWL (Jones Wilkins Lee) EOS for high explosives [21]. The relevant parameters are shown in Table I.

$$
P=A\left(1-\frac{\omega}{R_{1} V}\right) E_{m}^{-R_{1} V}+B\left(1-\frac{\omega}{R_{2} V}\right) E_{m}^{-R_{2} V}+\frac{\omega E_{0}}{V}
$$

where $A, B, R_{1}, R_{2}$, and $\omega$ are constants pertaining to the explosive, $V$ is the relative volume, $E_{0}$ is the initial energy per initial volume, $E_{m}$ is the material energy, $\rho$ is the mass density, $v_{D}$ is the detonation velocity, and $P_{C J}$ is the Chapman-Jouget pressure.

TABLE I: MAIN PARAMETERS OF C-4 EXPLOSIVE

\begin{tabular}{|c|c|c|c|c|}
\hline$\rho\left(\mathrm{g} / \mathrm{cm}^{3}\right)$ & $v_{D}(\mathrm{~cm} / \mu \mathrm{sec})$ & $P_{C J}($ Mbar $)$ & $A($ Mbar $)$ & B(Mbar $)$ \\
\hline 1.601 & 0.8193 & 0.28 & 5.0977 & 0.1295 \\
\hline$R_{1}$ & $R_{2}$ & $\omega$ & $V$ & $E_{0}\left(\mathrm{~J} / \mathrm{m}^{3}\right)$ \\
\hline 4.5 & 1.4 & 0.25 & 1.0 & 0.09 \\
\hline
\end{tabular}

\section{Air Parametes and Equation of State}

This study used the MAT_NULL material model to simulate air, and equations of state EOS_LINEAR_POLYNOMIAL were used to describe the material model as in (8) [19]. The relevant parameters are given in Table II.

$$
P=C_{0}+C_{1}+C_{2} \mu^{2}+C_{3} \mu^{3}+\left(C_{4}+C_{5} \mu+C_{6} \mu^{2}\right) E_{0}
$$

where $E_{0}$ is the internal energy per initial volume, $\mu$ is the coefficient of dynamic viscosity, $V$ is the relative volume, $C_{0}, C_{1}, C_{2}, C_{3}, C_{4}, C_{5}, C_{6}$ are constants, $\rho_{0}$ is the initial value of air density, and $\rho$ is the current air density.

TABLE II: MAIN PARAMETERS OF AIR

\begin{tabular}{cccc}
\hline \hline \multicolumn{2}{c}{$\rho\left(\mathrm{g} / \mathrm{cm}^{3}\right)$} & $E_{0}\left(\mathrm{~J} / \mathrm{m}^{3}\right)$ & $V\left(\mathrm{~g} / \mathrm{cm}^{3}\right)$ \\
\hline \multicolumn{1}{l}{$C_{0}$} & $2.5 \times 105$ & 1.0 & \\
\hline \multicolumn{1}{c}{$C_{0}$} & $C_{4}, C_{5}$ & $C_{1}, C_{2}, C_{3}, C_{6}$ \\
\hline \hline
\end{tabular}

\section{Soil Material Parametes}

The selection of soil composition should take into account the material porosity and its crushing or compacting behavior. The soil composition model of this study was also called Simple Elastic-Plastic [22]. Taking isotropic plasticity theory as the starting point, there is compressible plasticity in the material. This model can be used in porous material, such as soil, rock and concrete. Based on the above description, the experimental parameters of [23] were used for analysis, and the relevant parameters are given in Table III.

TABLE III: MAIN PARAMETERS IN THE SOIL MODEL

\begin{tabular}{cccc}
\hline \hline$\rho\left(\mathrm{g} / \mathrm{cm}^{3}\right)$ & $G(\mathrm{MPa})$ & $K_{u}(\mathrm{MPa})$ & $a_{0}$ \\
\hline 1.8 & 0.000639 & 0.3 & $3.4 \times 10-13$ \\
\hline$a_{1}$ & $a_{2}$ & \multicolumn{2}{c}{$P_{\text {cut }}(\mathrm{MPa})$} \\
\hline $7.03 \times 10-7$ & 0.3 & $-6.9 \times 10-8$ \\
\hline \hline
\end{tabular}

where $\rho$ is the mass density, $G$ is the shear modulus, $K_{u}$ is the bulk modulus, $a_{0}, a_{1}, a_{2}$ are yield function constant for plastic yield function below, and $P_{c u t}$ is the pressure cutoff for tensile fracture.

\section{RESULTS AND DISCUSSION}

Concerning the influences that blast vibrations have on slope stability, the sliding force primarily increases with the effects of vibrational inertial force. This is followed by a reduction in the structure's surface shear strength affected by repeated vibration loads, reducing the safety coefficients of the slope. For the physical quantities of the blast-induced vibration strength, the particle vibration velocity, acceleration, and shift were primarily analyzed. This study primarily analyzed the influences that horizontal shift quantity and vertical surface acceleration have on slope stability.

\section{A. Numerical Analysis Result and Empirical Equation Verification}

This study adopted the US-military TM5-1300 specifications as a reference for numerical analysis comparison. This technical specification is an empirical equation regarding the surface blast-induced wave propagation behavior summarized from the detonation experiment data to verify the reliability of the simulation analyses. To understand the propagation properties of the blast waves, this study separately investigated the peak blast pressure values at different horizontal distances from the detonation center $(50,100,200,300,400$, and $500 \mathrm{~cm})$.

Fig. 4 shows the relationships between the blast pressure duration curves. Figure 5 shows the attenuation trend between the numerical analysis and the peak blast pressure horizontal distance of the TM5-1300 empirical equation, and further illustrates the increase of the peak blast pressure values along with the decrease of prorogation distances. Concerning the analysis and comparison between the simulation results and the empirical equation based on US military specifications, this study found that the value range of the empirical equation was greater than the numerical analysis results. In addition, the differences within $100 \mathrm{~cm}$ from the detonation source were the most apparent. The blast pressure differences for within $50 \mathrm{~cm}$ and $100 \mathrm{~cm}$ from the detonation source were 7.036 MPa and 2.221MPa, respectively. 


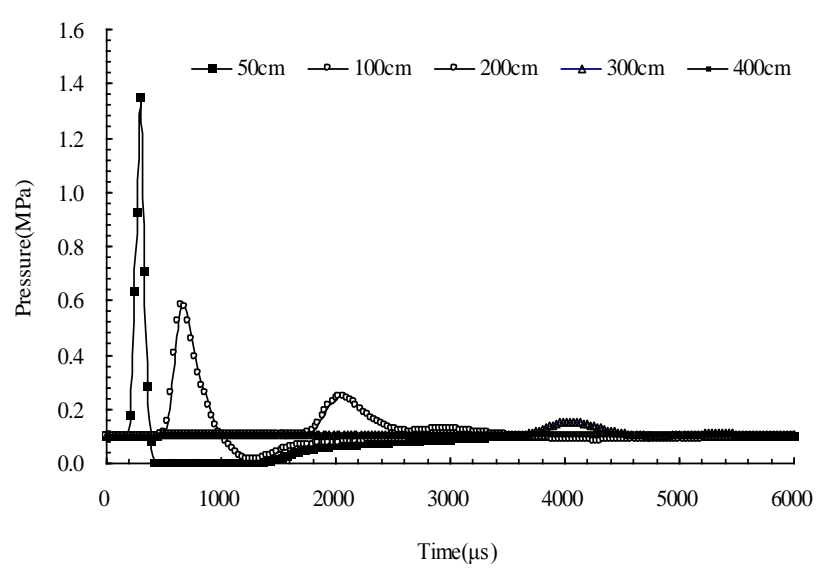

Fig. 4. Pressure-time histories curves under different distances condition.

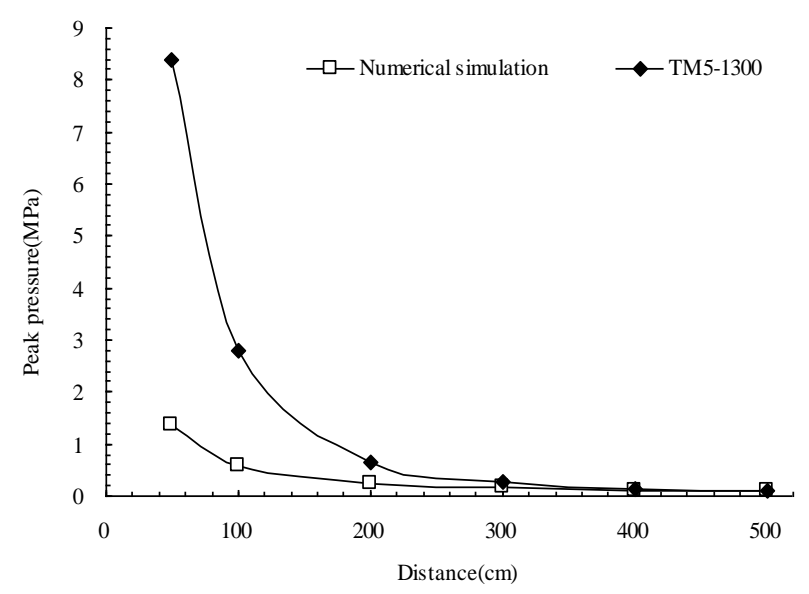

Fig. 5. Comparison of the peak pressure in the numerical simulation and TM5-1300 empirical.

The reason for the differences may be because of the varying environmental media during the experiments. However, considering the overall trend, these values comply with the attenuation properties of the blast wave energy; thereby confirming the reliability of the proposed analytical model. These results were then used to further investigate the influences that blast-induced seismic effects have on slope stability.

\section{B. Gradient Influences}

For the gradient influence analysis, the slope height of the proposed model was fixed at $300 \mathrm{~cm}$, and the gradients were set at $30^{\circ}, 45^{\circ}, 60^{\circ}$, and $75^{\circ}$. Observation points were established at the toe and top of the slope to investigate the influences that horizontal shift quantity and vertical surface acceleration have on slope stability.

Fig. 6 and Fig. 7 separately illustrate the horizontal shift relationships at the toe and top of the slope at different gradients. The diagrams indicate that the horizontal shift at the toe of the slope was greater than that at the top of the slope, and that the shift quantity gradually decreased with the increase in gradient, except for the gradients between $45^{\circ}-60^{\circ}$, which exhibited a gradual increase.

Fig. 8 and Fig. 9 separately illustrate the vertical surface acceleration relationships at the toe and top of the slope at different gradients. Analytical results show that the vertical surface acceleration was greater at the top of the slope than that at the toe, and that the vertical surface acceleration at the toe of the slope gradually increased with an increase in slope gradient; however, a gradual reduction was exhibited at gradients above $60^{\circ}$. In addition, the vertical surface acceleration at the top of the slope gradually decreased with the increase in slope gradient.

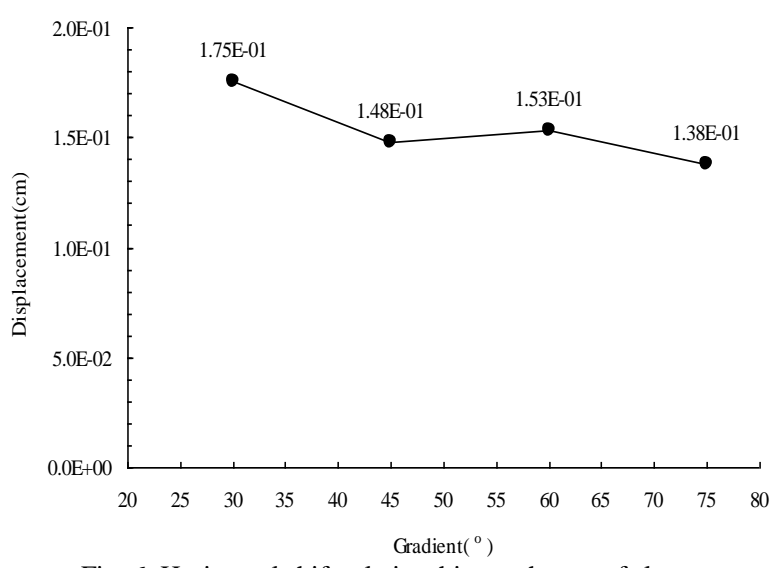

Fig. 6. Horizontal shift relationships at the toe of slope.

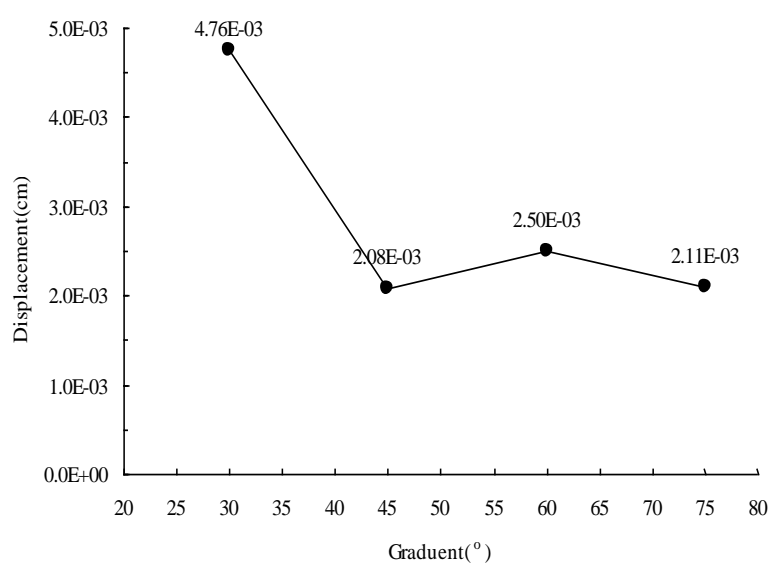

Fig. 7. Horizontal shift relationships at the top of slope.

These numerical analysis results show that, with increasing slope gradients, the horizontal shift at the toe and top of the slope gradually deceased; however, when achieving a specific gradient $\left(45^{\circ}\right)$ and within a specific gradient $\left(45^{\circ}-60^{\circ}\right)$, the horizontal shift indicated a gradual increase. A reduction in vertical surface acceleration at the toe was exhibited when the gradient increased to $60^{\circ}$. Thus, it was determined that the key gradient of soil slopes for blast-induce seismic effects was $60^{\circ}$. Construction designs should avoid the formation of steep slopes to reduce the effects of blast loads on slopes.

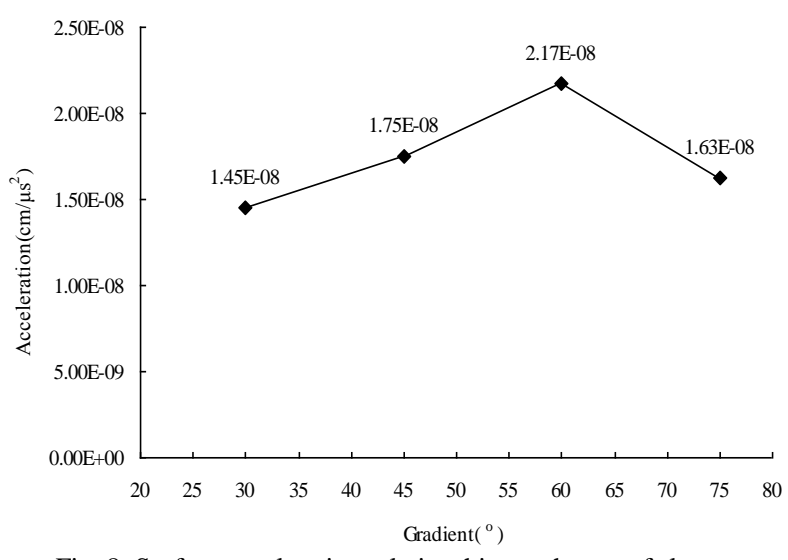

Fig. 8. Surface acceleration relationships at the toe of slope. 


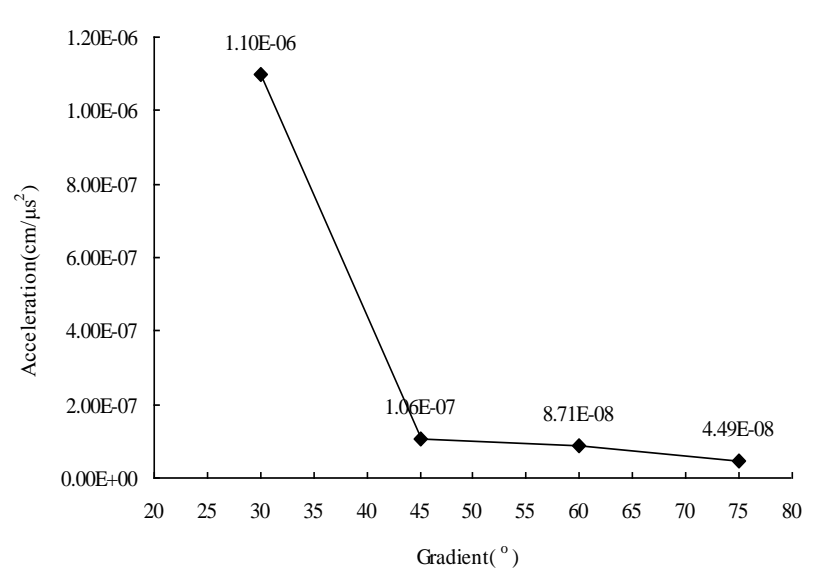

Fig. 9. Surface acceleration relationships at the top of slope.

\section{Height Influences}

For height influence analysis, the gradient for the model design was fixed at $45^{\circ}$, and the height was separately adjusted to $100,150,200,250$, and $300 \mathrm{~cm}$. Observation points were established at the toe and top of the slope to analyze the influences that horizontal shift quantity and vertical surface acceleration have on slope stability. Fig. 10 and Fig. 11 separately illustrate the horizontal shift relationships of the toe and top of the slope at varying heights. The figure illustrating the toe of the slope suggests that the horizontal shift gradually increases when slope height was less than $200 \mathrm{~cm}$ and gradually decreases when greater than $200 \mathrm{~cm}$. In addition, increasing slope height caused the horizontal shift at the top of the slope to gradually decrease; however, when slope height achieved a specific height (approximately $200 \mathrm{~cm}$ ), the changes in horizontal shift became smaller.

Fig. 12 and Fig. 13 separately illustrate the vertical surface acceleration relationships at the toe and top of the slope at varying heights. The figures suggest that the vertical surface acceleration at the top of the slope is greater than that at the toe, further indicating that the vertical vibration velocity peak value at the top of the slope was greater than that of the toe. This suggests that the vertical vibration velocity at the top of the slope possessed the amplification effect. When the height increased, the vertical surface acceleration at the toe of the slope gradually decreased. No reduction was exhibited when the height achieved the $150 \sim 200 \mathrm{~cm}$ range; however, the gradual reduction continued when the height exceeded $200 \mathrm{~cm}$. In addition, the vertical surface acceleration at the top of the slope gradually increases following the increase in slope height. At slope heights of 100,150,200, 250, and $300 \mathrm{~cm}$, the vertical surface accelerations $\left(\mathrm{cm} / \mu \mathrm{s}^{2}\right)$ at the top of the slope were 6.53E-08, 9.70E-08, 1.06E-07, 9.18E-08, and 8.72E-08 respectively. These results suggest that blast waves simultaneously possess both amplification and attenuation effects during the propagation process. Based on the numerical model analyzed in this study, when the slope is smaller than the critical height of approximately $200 \mathrm{~cm}$, the amplification effect is primarily produced; conversely, when the slope height exceeds $200 \mathrm{~cm}$, the attenuation effect is subsequently produced.

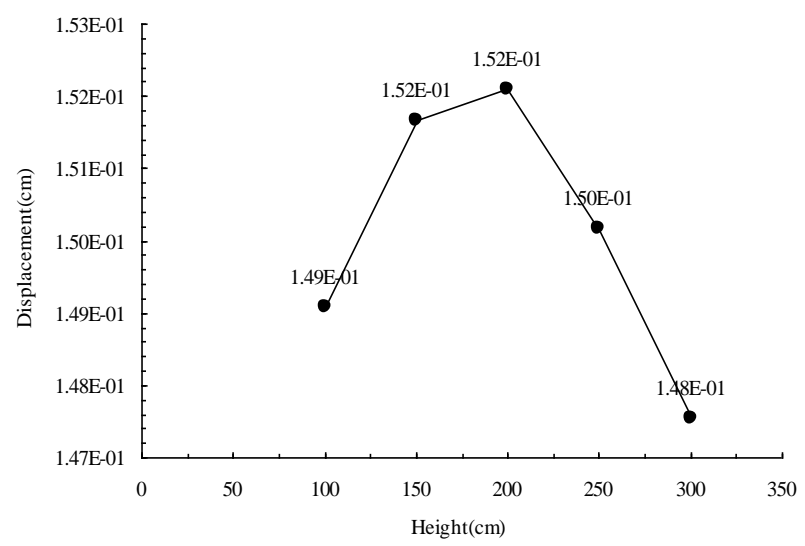

Fig. 10. Horizontal shift of the toe of slope at varying heights.

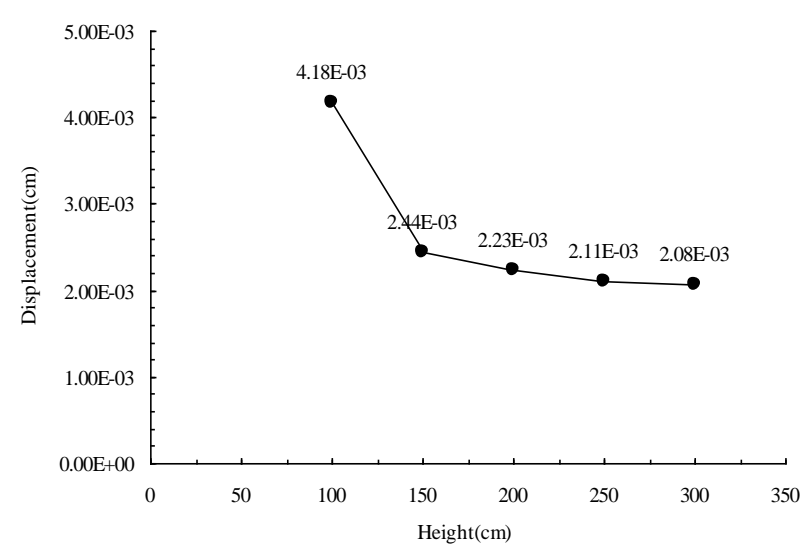

Fig. 11. Horizontal shift of the top of slope at varying heights.

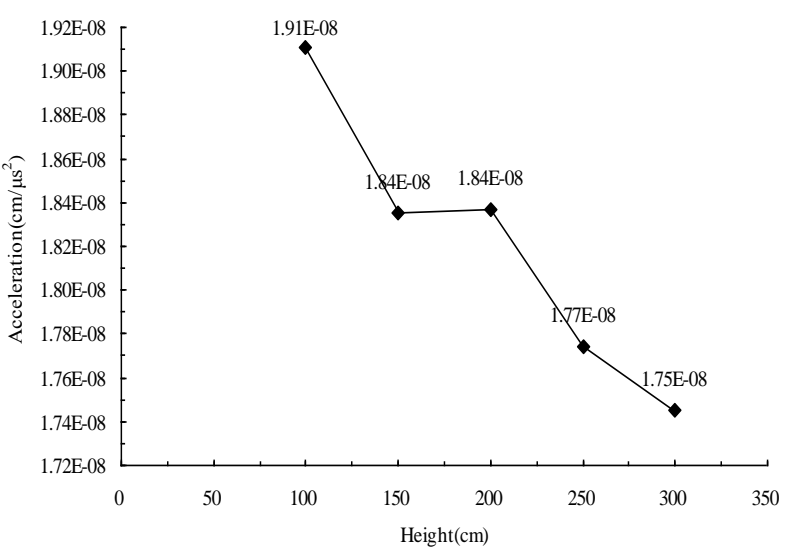

Fig. 12. Surface acceleration of the toe of slope at varying heights.

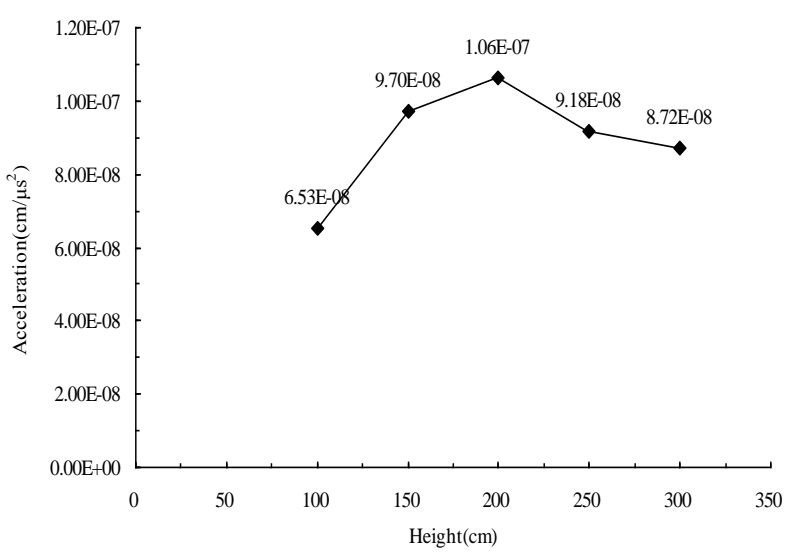

Fig. 13. Surface acceleration of the top of slope at varying heights. 


\section{CONCLUSION}

The propagation of blast waves is primarily based on media properties and blast design parameters. This study adopted a numerical simulation method to investigate the influence that blast-induced seismic waves have on the soil properties of slopes. In addition, the horizontal shift quantity and vertical surface acceleration of the slope were analyzed based on the changes in slope height and gradient to investigate the dynamic responses of the slope under blast conditions. The analytical results indicated that the key gradient for blast-induced seismic effects was $60^{\circ}$ and the blast waves simultaneously possess amplification and attenuation effects during the propagation process. The critical height for soil slopes was approximately $200 \mathrm{~cm}$ and the vertical vibration velocity peak value was greater at the top of the slope than at the toe.

This suggests that the amplification effect is present in the vertical vibration velocity at the top of the slope. It can be inferred from these results that the instantaneous blast-induced shock produces particle vibration propagation combined with acceleration under instantaneous shock wave effects, inducing dynamic loads and consequently causing the shear stress of the slope body to increase. After the blast vibrations produce compressive waves and transmit them onto the slope surface, this causes the slope surface to laterally shift and consequently induces the original discontinuous surfaces to expand and produce new discontinuous surfaces, which directly or indirectly influences slope stability. Thus, the influences that blast loads have on the dynamic responses of slopes must first be confirmed prior to construction planning and design, thereby reducing hazards caused by blast-induced ground vibrations and subsequently achieving the goal of maintained slope stability.

\section{ACKNOWLEDGMENT}

This study was partially supported by the National Science Council in Taiwan R.O.C. under through Grant NSC 102-2218-E-145-001.

\section{REFERENCES}

[1] Y. Koga and O. Matsuo, "Shaking table tests of embankments resting on liquefiable sandy ground," Soils and Foundations, vol. 30, no. 4, pp. 162-174, 1990.

[2] B. M. Das, Fundamentals of Soil Dynamics, U.S.A., 1982, pp. 122-152.

[3] Z. Q. Wang, H. Hao, and Y. Lu, "A three-phase soil model for simulating stress wave propagation due to blast loading," International Journal for Numerical and Analytical Methods in Geomechanics, vol. 28, no. 1, pp. 33-56, 2004.

[4] Z. Q. Wang and Y. Lu, "Numerical analysis on dynamic deformation mechanism of soils under blast loading," Soil Dynamics and Earthquake Engineering, vol. 23, pp. 705-714, 2003.
[5] C. C. Shen, M. W. Tang, and Y. C. Ding, "Influences of blast operations in the mining area of Eastern Taiwan have on slope and moldering wall stability," in Proc. Taiwan Rock Engineering Symposium, Tainan, June 2006, pp. 337-346.

[6] U. Longerfors, H. Westerberg and B. Kihlstrom, Ground Vibration in Blasting, Water Power, 1958, pp. 335-421.

[7] B. Baker and M. Garber, "Theoretical analysis of the stability of slopes," Geotechnique, vol. 28, no. 4, pp. 395-411, 1978.

[8] H. D. Charles, "Dynamic stability of rock slopes and high frequency traveling waves," Journal of Geotechnical Engineering, vol. 114, no. 10, pp. 1069-1088, 1988.

[9] M. Guowei, H. Hong, and Z. Yingxin, "Assessment of structure damage to blasting induced ground motions," Engineering Structures, vol. 22, pp. 1378-1389, 2000.

[10] M. S. Chafi, G. Karami, and M. Ziejewski, "Numerical analysis of blast-induced wave propagation using FSI and ALEmulti-material formulations," International Journal of Impact Engineering, vol. 36, pp. 1269-1275, October 2009.

[11] W. Lu, J. Yang, M. Chen, and C. Zhou, "An equivalent method for blasting vibration simulation," Simulation Modelling Practice and Theory, vol. 19, pp. 2050-2062, June 2011.

[12] K. Spranghers, I. Vasilakos, D. Lecompte, H. Sol, and J. Vantomme, "Numerical analysis and experimental validation of the dynamic response of aluminum plates under free air explosions," International Journal of Impact Engineering, vol. 54, pp. 83-95, November 2013.

[13] Technical Manual TM5-1300, "Structures to resist the effects of accidental explosions," Department of the Army, 1990.

[14] LS-DYNA Version 971 User's Manual, Livermore Software Technology Corporation, 2007.

[15] D. J. Benson, "Computational methods in Lagrangian and Eulerian Hydrocodes I," Computer Methods in Applied Mechanics and Engineering, vol. 99, issue 2-3, pp. 235-394, 1991.

[16] M. A. Puso, J. Sanders, R. Settgast, and B. Liu, "An embedded mesh method in a multiple material ALE," Computer Methods in Applied Mechanics and Engineering, vol. 245-246, pp. 273-289, August 2012.

[17] D. J. Benson, "Computational methods in Lagrangian and Eulerian Hydrocodes II," Dept. of AMES R-011 University of California, San Diego La Jolla, CA 92093, 1991.

[18] K. J. Bathe, Finite Element Procedures, Englewood Cliffs, Prentice Hall, 1996.

[19] LS-DYNA Theory Manual. Livermore Software Technology Corporation, 2006.

[20] LS-DYNA Theoretical Manual. Livermore Software Technology Corporation, 1998.

[21] B. M. Dobratz, LLNL Explosive Handbook Properties of Chemical Explosives and Explosive Simulants, Lawrence Livemore National Laboratory, 1981.

[22] W. F. Chen, Nonlinear Analysis in Soil Mechanics: Theory and Implementation, Amsterdam, New York Elsevier, 1990.

[23] J. Wang, Simulation of Landmine Explosion Using LS-DYNA 3D Software: Benchmark Work of Simulation of Explosion in Soil and Air, DSTO Aeronautical and Maritime Research Laboratory, DSTO-TR-1168, 2001.

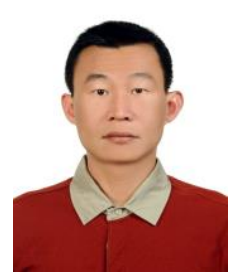

Iau Teh Wang received his Ph.D. degree from Department of Civil Engineering, Graduate Institute of Disaster Prevention on Hillslopes and Water Resources Engineering, National Pingtung University of Science and Technology, Taiwan, R.O.C. in 2013 Presently, he is an assistant professor of the Department of Civil Engineering, Chinese Military Academy, Taiwan, R.O.C., from 2007 till now.

His research areas include explosion mechanics, dynamic finite element methods, slope stability analysis, landslides control engineering, etc. 